\title{
Fluchtpunkt Vergangenheit - Erinnerungskultur in Bulgarien nach dem Ersten Weltkrieg
}

\author{
CLAUDIA WEBER (Leipzig)
}

Nach der Niederlage im Ersten Weltkrieg war Bulgarien in einem desolaten Zustand. Der Friedensvertrag von Neuilly hatte das Staatsgebiet um fast 9000 $\mathrm{km}^{2}$ reduziert. Mit dem Zugang zur Ägäis und der Dobrudža verlor Bulgarien zentrale Wirtschaftsräume, die nicht nur bei der Versorgung der über 200.000 Flüchtlinge schmerzlich fehlten. Die Monarchie war diskreditiert und das traditionelle Parteiensystem erschüttert. Am 3. Oktober 1918 hatte Zar Ferdinand zugunsten seines Sohnes Boris abgedankt und war nach Deutschland zurückgekehrt. Die ersten Nachkriegswahlen im August 1919, bei denen die Kommunisten und Sozialdemokraten zusammen auf 31 Prozent der Wählerstimmen kamen, besiegelten die Erosion der bulgarischen Vorkriegsgesellschaft. ${ }^{1}$ Schlimmer noch als die politische Krise, die ökonomischen Einbußen und die das Land auf Jahre hinaus belastenden Reparationsleistungen aber wog das Kriegstrauma auf einer Gesellschaft, die sich um ihre nationalen Rechte betrogen fühlte und stattdessen mit Tod und Trauer konfrontiert war. Die über 160.000 meist auf den Schlachtfeldern des Weltkrieges gefallenen Bulgaren hinterließen eine zutiefst verunsicherte Gemeinschaft, die den Umgang mit dem Erlebten ebenso erlernen musste, wie sie ein zukunftsfähiges Selbstverständnis benötigte. ${ }^{2}$

Der folgende Artikel erzählt von den historischen Mythen und Vergangenheitsdeutungen, mit deren Hilfe die politische Elite die Nachkriegskrise bewältigen wollte. Dabei wird einerseits nach der sozialen Wirkungsmacht und öffentlichen Akzeptanz dieser Geschichtskonstruktionen gefragt. Andererseits werden sie in den Kontext einer nationalen bulgarischen Erinnerungskultur seit der Nationalstaatsgründung 1878 gestellt, um Unterschiede und Gemeinsamkeiten zu früheren Deutungsangeboten aufzuzeigen. In einem zweiten Teil geht es dann um die symbolische Verarbeitung der Kriegserfahrungen, wobei vergangenheitspolitische Strategien ebenso untersucht werden, wie die Rituale und Deutungsmuster einer in den lokalen Räumen verorteten Kriegserinne-

1 Zur Ereignisgeschichte in: Crampton 1993, 82ff; Rothschild 1990, 323ff.

2 Vgl. zu den Deutungsmustern des Ersten Weltkrieges in Europa u.a. in: Winter 1995; Ders. 1988; Ders. 1999; Mosse 1990; Fussell 1975; Hirschfeld / Langewiesche / Krumeich 1997. 
rungskultur. Die Untersuchung beider, des Umgangs mit den Geschichtsmythen ebenso wie der symbolischen Strategien der Kriegserinnerung, erscheinen insbesondere vor dem Hintergrund des bereits angedeuteten politischen und gesellschaftlichen Umbruchprozesses interessant, war doch in Bulgarien im Mai 1920 mit dem Bulgarischen Nationalen Bauernbund (bălgarski zemedelski naroden săjuz - BZNS) unter Aleksandăr Stambolijski eine politische Kraft an die Macht gelang, die zu den Kriegsgegnern gehört hatte und die ein radikales innen- und außenpolitisches Reformprogramm propagierte. Neben der Bewertung der symbolischen Vergangenheitspolitik der neuen Regierung verspricht der Artikel so auch Einblicke in die Wirkungsmacht nationaler Geschichtsmythen als gemeinschaftsstiftende Phänomene der longue dureé.

Obwohl an dieser Stelle nicht auf die gesellschaftliche Vision Stambolijskis und die historische Bewertung seiner Reformen eingegangen werden kann, erscheint es gerade im Hinblick auf die Vergangenheitspolitik des Bauernbundes unabdingbar, auf den außenpolitischen Ansatz und den innenpolitischen Umgang mit den vermeintlichen Kriegsschuldigen hinzuweisen. ${ }^{3}$ Um Bulgarien aus der „nationalen Katastrophe“ der Kriegsniederlage herauszuführen, bewies der Bauernbund einen durchaus beeindruckenden Willen zur politischen Neugestaltung. Außenpolitisch bedeutete dies vor allem die Absage an eine großbulgarische Nationsidee und die Verbesserung des serbischbulgarischen Verhältnisses bis hin zu Bemühungen um den Aufbau einer „Grünen Internationale“ und zukünftigen Balkanföderation. Die damit verbundene Aufgabe territorialer Ansprüche auf Makedonien machte Stambolijski nicht nur in den Augen seiner politischen Feinde und der IMRO (Innere Makedonische Revolutionäre Organisation) zu einem „Vaterlandsverräter“, er brüskierte auch einen Großteil der Bevölkerung, für die Makedonien auch nach der Kriegsniederlage ein Teil der historisch-nationalen Identität blieb.

Innenpolitisch sorgte Stambolijski für die strafrechtliche Verfolgung der früheren Kriegsregierung. Im Dezember 1919 trat das „Gesetz zur Verurteilung der Schuldigen an der nationalen Katastrophe" in Kraft, mit dessen Hilfe die Mitglieder der Regierung Radoslavov und weitere Personen, die unmittelbar an der Kriegserklärung beteiligt gewesen waren, zum Tod oder zu lebenslanger Haft verurteilt wurden. Gefängnisstrafen erwarteten jene, die nur mittelbar, im Parlament oder als Diplomaten an der Kriegsvorbereitung gewirkt hatten. Mit der juristischen Schuldzuweisung an die Vorkriegselite waren, stellvertretend für alle Bulgaren, die den „nationalen Traum“ mitgeträumt hatten, die Sündenböcke gefunden. Die politische Externalisierung der Kriegsschuld war eine Bewältigungsstrategie, aber kein Deutungsangebot, das der Kriegserfahrung langfristig hätte Sinn verleihen können. Um dieses Deutungsangebot soll es im Folgenden gehen.

3 Als gültige Monografie zur bulgarischen Bauernpartei und ihres Führers gilt auch noch nach über zwanzig Jahren: Bell 1977. Siehe weiterhin: Božkov / Ninov 1980; Stambolijski 1980. 
So ernst der Bauernbund die politische Abkehr von irredentistischen Großmachtträumen erklärte, so sehr beschreiben die Rede von der Umkehr, von der Politik der ,friedlichen Beziehungen auf der Balkanhalbinsel“" und das Verdammen des Nationalismus als „Geißel der Balkanvölker“ nur die eine Seite der Stambolijski-Jahre. ${ }^{4}$ Zwischen politischer Programmatik und Realität klaffte eine Lücke, die gerade bei der Betrachtung der Vergangenheitspolitik und Erinnerungskultur deutlich wird und die von der historischen Forschung mit ihrer bisher starken Konzentration auf die politische Geschichte jener Jahre nur unzureichend beachtet worden ist. Die andere Seite der Politik Stambolijskis offenbart einen erstaunlich offenen und kontinuierlichen Umgang mit traditionellen Deutungsmustern des Nationalen, wie er zum Beispiel im Umgang mit dem historischen Mythos der Slawenapostel Kyrill und Method zutage trat.

\section{Die Kyrill- und Method-Feiern 1921}

Während der Kriege hatten die Erinnerungen an Kyrill und Method zur Legitimation territorialer Expansion gedient. Nun, nach der Niederlage, standen sie für eine tröstende ruhmreiche Kulturgeschichte und die Überlebensfähigkeit des Bulgarentums nach den Wirren der Weltgeschichte. „Auf diesem starken Bewusstsein", hieß es in der Festzeitung des Bildungsministeriums zum Kyrillund Method-Tag 1921,

und auf der moralischen und kulturellen Qualität unseres Daseins müssen wir das Gebäude der heutigen Zeit errichten [...], wir müssen in unseren Seelen das Schutzschild zur Bewahrung unserer Nationalität aufrichten. [...] Unsere Kraft sie ist in unseren Seelen. Das Licht, das uns durch den düsteren Weg des Lebens leiten wird - es ist uns seit Jahrhunderten erhalten durch die Heiligen Brüder. Mit ihm und dem Feuer unserer Seelen werden wir die Schwäche besiegen. ${ }^{5}$

Zur Verbreitung dieses Orientierungsangebotes bediente sich die Bauernpartei bedenkenlos des alten Materials der Kriegspropaganda. Schon in der ersten Ausgabe der ab 1921 vom Bildungsministerium jährlich geplanten Kyrill-und Method-Gedenkschrift erschien ein nur leicht veränderter Neuaufguss der 1916 in der Propagandazeitschrift Otečestvo publizierten Erzählung Elin Pelins. ${ }^{6}$ In den Kriegsjahren hatte die Geschichte von der versteckten Ikone das antiserbische Feindbild untermauert und ein unbesiegbares bulgarisches Nationalbewusstsein in Makedonien beschworen. Fünf Jahre später war „Bulgarische Beschützer", so der Titel der Erzählung, zwar nicht mehr im makedonischen

4 Zemedelska Bălgarija II (1922) 119, 1.

5 Sv. sv. Kiril i Metodij 24/11 maj 1921 god. 1921, 3.

6 In der Festzeitung veröffentlichten Autoren, die während des Krieges zu Stammautoren von Otečestvo gehört hatten. Elin Pelin, „Bălgarski zakrilnici“, in: ebd., $12 f$. 
Ochrid, sondern im geografischen Niemandsland angesiedelt, und der Feind war namenlos, die Moral aber blieb gleich. Wieder überstand das bulgarische Volk - versinnbildlicht in der geretteten Ikone - die Katastrophen der Geschichte.

Der 24. Mai, der Tag Kyrills und Methods und des bulgarischen Schrifttums, wurde nach dem Krieg zum Feiertag nationaler Einheit und Rekonvaleszenz, zum Pflaster für die tiefen Wunden einer auseinanderklaffenden Nation. Im Vorfeld der Feiern appellierte das Bildungsministerium wiederholt an die Bevölkerung, mit diesem „,bulgarischen Feiertag seiner großen Idee stärksten Ausdruck zu geben, [...] der Idee innerer moralischer Einheit“". „,Ein Volk wie das unsere", verbreitete Bildungsminister Stojan Omarčevski einen vergangenheitsgesättigten Optimismus,

das in seiner Entwicklung noch schrecklichere Katastrophen aushielt [...] als die heutige, verlor nie und wird den Glauben an eine bessere Zukunft nicht verlieren. [...] Ein Volk, das in seiner Geschichte so epochale Taten wie die Kyrills und Methods aufzuweisen hat [...], so ein Volk, möge auch sein Unglück noch so groß sein, wird die Kräfte zur Überwindung finden. ${ }^{8}$

Wie kaum ein anderer Erinnerungskult war der um Kyrill und Method mit einer kollektiven Selbstvergewisserung verbunden, die auf den Codes „Kultur“, „Bildung“ und „Schrifttum“ basierte. Mit den Gedenkfeiern 1921 knüpfte die Bauernpartei an die tradierte Erzählung von nationalem Stolz und Selbstbewusstsein an. Das Bildungsministerium sandte symbolische „Streicheleinheiten“ an das Volk und stilisierte die Slawenapostel zum Ursprung kultureller Größe:

Wir, die Bulgaren, nahmen mit dem Wirken unserer Apostel den christlichen Glauben an und schufen ein großartiges Schrifttum, wir waren fähig, die Strahlen des Lichtes in die slawischen Länder zu leiten. ${ }^{9}$

Viele der in der offiziellen Festschrift erschienenen Artikel hatten denselben Tenor. Wenn auch die gesamtslawische Bedeutung der Leistungen Kyrills und Methods stets betont wurde, hätten sie doch in Bulgarien ihren Ursprung gehabt. Bulgarien, die „Morgenröte der slawischen Kultur“, so der Autor Dimităr Mišev, hätte, als es die Apostel aufnahm und deren Schrifttum verbreitete, den Erfolg der Slawenmission ermöglicht. ${ }^{10}$ Die auf die kulturhistorische Größe Bulgariens abzielende Ausdeutung der Kyrill- und Method-Geschichte entsprach dem staatlichen Anliegen, Kriege und militärischen Ruhm als Quellen nationalen Stolzes durch Kultur, Bildung und Sprache zu ersetzen. Kyrill und Method waren Symbole für den

7 Učilišten pregled XX (1921) 6, 7, $294 f$.

8 Tadžer 1922, 134.

9 Ebd., 133.

10 Mišev 1921, $9 f$. 
Geist der Bulgaren - gesund, lebhaft, kraftvoll, von bösen [...] Plagen und unerträglichen Katastrophen oft behindert, aber immer das Heilige in der Seele bewahrend - seine Sprache und seinen Stamm (roda si). ${ }^{11}$

Für die Bauernpartei waren Kultur und Bildung die neuen Codewörter für einen Nationalstolz, auf den sich das Volk nun besinnen müsse. „Der einzige [...] Stolz unserer Seele ist unser Streben nach Bildung und Kultur, uns hinterlassen von den Heiligen Brüdern“, verkündete das Bildungsministerium und beschwor die Erinnerungen an die große Nationalkultur als Medizin gegen die eigene und fremde Kriegslust:

Das ist unser Schutz gegen die barbarischen Instinkte der neidischen, [...] unersättlichen Völker nach fremder Erde [...]. Es ist unbestritten, dass Kultur die Barbarei zerstören wird, dass Licht die Dunkelheit vertreibt und das Gute wiederauferstehen wird, denn die Wahrheit ist ewig. ${ }^{12}$

Die „Kultur“, mit der die Bauernpartei „die barbarischen Instinkte“ besiegen wollte, war jedoch keine andere als die der geheiligten Nation. Ihr Kulturnationalismus verkörperte sowohl den Bruch mit der militaristischen Vergangenheit und doch gleichzeitig die Kontinuität ihrer großbulgarischen Erinnerungsmuster. Der durchaus genuine Glaube an die erzieherische Wirkung von Bildung kulminierte - gepaart mit der Suche nach einheitsstiftenden Identitätsangeboten - in der mythischen Überhöhung nationaler Kulturtradition. Kyrill und Method, Kultur und Bildung erlangten nach dem Krieg einen fragwürdigen Kultstatus, mit dem sich die Reformer in jenes Fahrwasser begaben, das sie eigentlich umschiffen wollten. Der alten Streitfrage nach der nationalen Zugehörigkeit der Apostel wich Omarčevski aus. Einerseits, betonte er in einer Festrede, seien Geburtsort und der „Name der Mutter“ von zweitrangiger Bedeutung, andererseits aber sei unbestritten, dass in den „Herzen der Heiligen Apostel eine gewisse Nähe (nešto blizko) zum bulgarischen Volk" ${ }^{\text {"13 }}$ vorherrsche. Teilweise derartig verbrämt, teilweise aber auch erstaunlich offen machte die neue Regierung Zugeständnisse an eine altbekannte Deutung kultureller Superiorität. „Durch die Jahrhunderte der Geschichte“, hieß es aus dem Bildungsministerium,

waren wir unter unseren Nachbarn die „Kulturreichsten“ (naj-kulturnite), wir waren auch unseren entfernteren Stammesverwandten Lehrer und Aufklärer [...], das bulgarische Wort, kraftvoll und inspirierend, machte sie zu Kulturvölkern. ${ }^{14}$

Die Zeremonien des 24. Mai, an dem, so Omarčevski, ,,wir den Namen und die Taten derer ehren, die uns das Augenlicht gegeben und unsere Seele er-

11 Ebd., 3.

12 Centralen dăržaven istoričeski archiv (weiter: CDIA), fond 177, op. 1, a. e. 625, list 47. Rundschreiben Nr. 10894 vom 19. April 1921.

13 Tadžer 1922, 133.

$14 C D I A$, fond 177 , op. 1, a. e. 625 , list 47. 
leuchtet haben", ${ }^{15}$ blieben größtenteils unverändert. Wie in den Jahrzehnten zuvor erteilte das Bildungsministerium auch 1921 die zentralen Vorgaben für Feiern mit ,wahrhaft nationalem Charakter“ ${ }^{16}$ Der Vortag war schulfrei und der Vorbereitung gewidmet, wobei das Ministerium mit der Anweisung, die Feiern an zentralen Plätzen der Gemeinden stattfinden zu lassen, besonderen Wert auf die Verbindung zwischen Schule und öffentlichen Raum legte. Von großer Bedeutung für den Volkscharakter der Feiern sei, so das Ministerium, das Erscheinen der Jugend in Trachten und Nationalkostümen. In Sofia veranstaltete die Universität am 23. Mai im Nationaltheater ihre Festveranstaltung, an der aufgrund der Streitigkeiten mit den Akademikern nicht Stambolijski, wohl aber der neue Zar Boris III. teilnahm. Ein Schülerchor trug Volkslieder vor, Schauspieler rezitierten Kyrill Christovs Bălgarskata reč (Bulgarische Rede), und Aleksandăr Teodorov-Balan, der zu den akademischen Steigbügelhaltern des Kriegsnationalismus gehört hatte, sprach zur Bedeutung der Slawenmission für Bulgarien. Der 24. Mai begann mit dem traditionellen Festgottesdienst in der Sofioter Kathedrale „Sv. Nedelja“. Angeführt von einer Ikone der Apostel marschierte der Festumzug zum „Nevski-Platz“, wo ein weiterer Gottesdienst unter freiem Himmel geplant war. Aus „einigen hundert Seelen“, so die Zeitungen am nächsten Tag, erklang die Hymne der Wiedergeburtsepoche Vărvi, narode văzrodeni. ${ }^{17}$ Unter Glockengeläut versammelten sich die Schüler der Hauptstadt, Studenten der Militärakademie, Vertreter von Kulturvereinen, aber auch die makedonischen Flüchtlingsvereine mit Trauerflaggen und die Verbände der Reserveoffiziere. Am Nachmittag begannen auf einer großen Festwiese am Stadtrand Turnvorführungen, die die Ausgelassenheit früherer Volksfeste durch Körperertüchtigung und Sport ersetzen sollten. Aufgeteilt in vier Gruppen, führten Schüler vor einem Pavillon mit den Statuen Kyrills und Methods ihre Gymnastikübungen vor. Am Abend lud das Bildungsministerium zu einem Festbankett ein, auf dem neben der neuen Regierung auch die alte Garde der Kriegsdichter von Kyrill Christov bis Ivan Vazov dem neuen Kulturnationalismus huldigte. „Niemals war Sofia einmütiger in seinen Gedanken und Gefühlen [...] wie am Tag der Heiligen Apostel, der als Tag der Erneuerung, Erfrischung und Wiedergeburt eingehen wird" ${ }^{18}$ beschrieb ein wohlwollender Chronist der Stambolijski-Jahre die versammelte Festgesellschaft. Die makedonischen Vereine, die Reserveoffiziere, große Teile der alten Elite, aber auch die Bevölkerung begrüßten das vertraute Anknüpfen an die gewohnten Riten, Symbole und Deutungen, das die Pflege eines erschütterten Selbstwertgefühls nicht nur förderte, sondern auch den politischen Wechsel verträglicher erscheinen lie $\beta$, als die offizielle Propaganda hatte vermuten lassen. Nur von den Kommunisten wurde diese vergangenheitspolitische Konti-

$15 S v$. sv. Kiril i Metodij, 1921, 3.

16 CDIA, fond 177 , op. 1 , a. e. 625 , list 47.

17 Tadžer 1922, 133.

18 Ebd., 136. 
nuität kritisiert. Seit Kriegsende ohnehin die schärfste Konkurrenz der Bauernpartei, lehnte sie den 24. Mai als „,bürgerlichen Feiertag“" grundsätzlich ab und rief zum Boykott auf. Stattdessen forderten sie, den 1. Mai, die „Rote Woche“ und den „Internationalen Tag der Jugend“ als neue, nichtnationale Feiertage einzuführen. ${ }^{19}$ Das Bildungsministerium konterte mit dem Vorwurf „,unbulgarischen Verhaltens“ der Kommunisten, die sich „leider leichter nach fremden Traditionen als in Richtung eines vollkommenen jahrhundertealten Gedankens“ orientieren würden. „Der 1. Mai“, so die Regierung, ,ist kein bulgarischer, sondern ein westeuropäischer [...] Feiertag" und gehöre von daher nicht in den Feiertagskanon. ${ }^{20}$ Das Bildungsministerium ging gegen die „Verunglimpfung des hellsten und schillerndsten Tages unseres historischen Lebens" ${ }^{\text {"21 }}$ mit handfesten Mitteln vor. Lehrer, die pro-kommunistischer Gedanken verdächtig waren oder sogar am 24. Mai 1921 Gegendemonstrationen organisiert und sich geweigert hatten, den Anordnungen des Ministeriums zu folgen, wurden entlassen.

\section{Kriegserinnerung nach der „nationalen Katastrophe“}

Das Trauma der Kriegsniederlage verlangte von der neuen Regierung nicht nur ein in der nationalen Vergangenheit verankertes kollektives Orientierungsangebot, sondern, damit einhergehend, ebenso dringlich die unmittelbare Auseinandersetzung mit den Erfahrungen der Gegenwart: mit dem Kriegstod, der Not der Hinterbliebenen und der kollektiven Verlusterfahrung. Die Umwandlung dieser existentiellen Erschütterungen in eine sinnhafte Narration zur kollektiven Rekonvaleszenz war die Nagelprobe für die Identitätspolitik der Bauernpartei, deren Handlungsspielräume begrenzt waren.

Seit der Staatsgründung im Jahr 1878 waren die Kriege in der bulgarischen Geschichte ausschließlich durch die nationale Optik betrachtet worden. Wie überall in Europa war der Krieg auch in Bulgarien der Katalysator der politischen und historisch-mythischen Nationsbildung gewesen. Er hatte die Gemeinschaft nach innen definiert und über Freund- und Feindzuweisungen nach außen abgegrenzt. Seitdem der russisch-türkische Krieg von 1877/78 im nationalen Gedächtnis als der große Befreiungsakt verankert war, dessen Sieg nur von der Schmach des Berliner Vertrages getrübt war, galten rückblickend alle der bulgarischen Nationalgeschichte zugeschlagenen Kriege als Belege für kollektive Charakterzüge von Heldenmut und Opferbereitschaft, ferner dienten Sie zur Legitimation vermeintlich historischer Territorialrechte. Sie erin-

19 Otčet na ministra na narodnoto prosveštenie Stojan Omarčevski za dejnostta na ministerstvoto ot 20 maj 1920 go 1 april 1921 god, 1921, 164. (Rechenschaftsbericht des Bildungsministers für die Zeit vom 20. Mai 1920 bis 1. April 1921), 6ff, 21.

20 Sv. sv. Kiril i Metodij, 1921, 4.

21 CDIA, fond 177 , op. 1, a. e. 625 , list 47. 
nerten an vergangenen Ruhm, aber auch an die zukünftige Pflicht, das großbulgarische San Stefano-Ideal zu erfüllen. ${ }^{22}$ Die Tradition, Kriege als Befreiungsakt oder Vereinigungskampf zu interpretieren, wurde zum Problem einer Partei, die sich offiziell der Abkehr vom großbulgarischen Traum verschrieben hatte und trotzdem nicht umhin kam, einen Sinn für das Sterben und die Gewalt zu finden. Theoretisch hatte die Bauernpartei verschiedene Möglichkeiten. Sie konnte dem Krieg jeglichen Sinn absprechen - eine angesichts der fundamentalen Zäsur vollkommen unrealistische Option. Und nur unmittelbar nach Kriegsende, in der von Ernst Troeltsch für das deutsche Beispiel beschriebenen Zeit des „Traumlandes“ nach der ersten Verliererdepression und in einer eigentümlichen Euphorie über die Absetzung des alten Regimes, existierte für die Agrarier die Chance, die Kriege als Befreiung nicht nur von der korrupten Politikerkaste, sondern auch von der Geißel des nationalen Ideals zu deuten. ${ }^{23}$

Die Bauernpartei entschied sich für eine ambivalente Version aus Kriegsrechtfertigung und Kriegsablehnung. Aus dem Ersten Weltkrieg wurde offiziell die „Erhebung des heldenhaften bulgarischen Volkes für seine nationale Einheit“, die, so Bildungsminister Omarčevski, „zur moralischen Erbauung der jungen Generation [...] und zur Stärkung eines warmen patriotischen Gefühls für ewig im nationalen Gedächtnis festgehalten “24 werden müsse. Andererseits sollten die Denkmäler, die zur Erinnerung an diese „Erhebung“ errichtet werden würden, an die „Dummheit der [...] Regierenden erinnern, die mit einem Krieg die nationale Vereinigung probierten. "25 Die Bauernpartei trennte zwischen den historischen Rechten auf nationale Vereinigung und einem von Regierung und Monarch angezettelten Krieg, in dem diese Rechte missbraucht worden waren. Sie verurteilte den Kriegsnationalismus und versuchte doch gleichzeitig, dem Tod einen nationalen Sinn zu verleihen.

Für die Entscheidung, den Krieg als volkspatriotischen Aufstand zu deuten, gab es verschiedene Gründe. So hatten der offizielle Widerruf bulgarischer „Rechte“ an Makedonien und die Unterzeichnung des serbisch-bulgarischen Vertrages am 23. März 1923 den Parteiführer Aleksandăr Stambolijski nicht nur in den Augen der alten Eliten und entlassenen Militärs zum Vaterlandsverräter gestempelt. Mit einer explizit national-historisch ausgerichteten Erinne-

22 Der Präliminarfrieden von San Stefano wurde am 19. Februar 1878 nach dem Sieg Russlands zwischen dem unterlegenen Osmanischen Reich und dem Zarenreich unterzeichnet. Er sah die Errichtung eines dem Osmanischen Reich weiterhin tributpflichtigen großbulgarischen Staates vor, dessen Grenzen von Makedonien bis an das Schwarze Meer und südlich bis an die Ägäis reichen sollten. Nach der Revidierung dieses von vornherein machtpolitisch unrealistischen Entwurfs durch die europäischen Großmächte auf dem Berliner Kongress im Juni 1878 musste sich Russland mit einem territorial erheblich verkleinerten Bulgarien abfinden.

23 Siehe ausführlich bei: Schivelbusch 2001, 21 f.

24 Omarčevski 1920, $157 f$.

25 Ebd. 
rungspolitik hoffte die Regierung einerseits, den Vorstellungen der Bevölkerung zu entsprechen, deren historisches Selbstverständnis sich stets in nationalen Kategorien und Wertemustern bewegt hatte. ${ }^{26}$ Andererseits war das Deutungsangebot der Agrarier darauf gerichtet, die durch die Niederlage mental gedemütigten und sozial deklassierten Militärs in eine Gegenwart zu integrieren, aus der sie zunächst ausgeschlossen schienen. Ihre Verbände wie die 1914 gegründete Militärliga, der Zentrale Wohltätigkeitsverband der Reserveunteroffiziere „Gurguljat“ oder der Verband der Reserveoffiziere, die ,allseitig gesellschaftlich aktiv“ für eine „Wiederbelebung des nationalen Ideals“ eintraten, waren in der Zwischenkriegszeit zu Auffangbecken nationalistischer Ressentiments geworden ${ }^{27}$ Mit einem Gefallenenkult, der aus den toten Soldaten, den Veteranen und Invaliden nationale Helden machte, demonstrierte die Bauernpartei Respekt, Anerkennung und nahm den Militärs, indem sie deren Deutungsmonopol verhinderte, den Wind aus den Segeln. Das Kriegsministerium kümmerte sich mit der Einrichtung des Fonds „Sv. Vrač“ nicht nur um die soziale Versorgung der „teuren Opfer“. Am 27. November 1919, dem Unterzeichnungstag des Neuilly-Vertrages, gegründet, sorgte ein weiterer Fond, „Našata konnica“ (Unsere Kavallerie), über den Verkauf von Broschüren, Gedenkblättern, Illustrierten, die Gründung eines Kavallerie-Museums und das Aufstellen von Denkmälern ,fuir das Gedenken und die Verbreitung der Kriegsheldentaten (bojnite podvizi) bulgarischer Kavalleristen “. ${ }^{28}$ Auch an der Militärakademie entstand 1921 ein Fond zum ,,ehrenden Gedenken an die gefallenen Offiziersabsolventen der Akademie“, die „für unser Volksblut teuren Opfer (skăpi za rodnata ni krăv žertvi)“. ${ }^{29}$

Im Dezember 1922 stellte die Regierung mit dem „Denkmal des unbekannten bulgarischen Soldaten “ ihr größtes Projekt der Kriegserinnerung vor. ${ }^{30}$ „Was haben wir Lebenden getan“, fragte der Kriegsminister Konstantin Tomov,

um zu zeigen, dass wir das größte Opfer, das sie [die Gefallenen, Anm. d. Verf.] gaben, wertschätzen? Womit rufen wir heutige und zukünftige Generationen zur Nachahmung auf? Womit bedanken wir uns? ${ }^{31}$

26 Dimitrova 2002, 1-9, hier 8.

27 Zlatnijat almanach na Bălgarija, 1938, 611.

28 Bălgarski voenen almanach, 1926, 661.

29 Ebd., 659f.

30 CDIA, fond 173, op. 3, a. e. 3185, list 2. Erklärung zum Gesetzesprojekt zur Errichtung des „Denkmals für den unbekannten bulgarischen Soldaten“ vom 20. Dezember 1922 (Motivi kăm zakonoproekt za văzdigane pametnik na „neznajnija bălgarski vojnik“"). Das Gesetz wurde am 24. Februar 1923 angenommen, nachdem es bereits im Dezember 1922 vom Ministerrat und dem Zaren befürwortet worden war. Der Staat stellte eine Million Leva zur Verfügung. Beim Kriegsministerium wurde ein Fond eingerichtet, nationale Spendenaufrufe und Veranstaltungsabende organisiert.

31 Ebd. 
Mit dem Denkmal entstand ein Ort, der das staatliche Deutungsangebot im öffentlichen Raum repräsentierte. „Die der Heimat teuren Opfer“, so Tomov in seiner Gesetzesbegründung,

steigerten die Ehre, den Ruhm und die Größe unseres Volkes. Sie bewiesen, dass der Bulgare zu Opfern bereit ist. Sie bewiesen, dass in der Tiefe des bulgarischen Herzens jene heilige Flamme verborgen ist, die ihm die Väter und Großväter übergaben, die heiligen Patrioten Levski, Botev, Karadža. ${ }^{32}$

Die Bauernpartei historisierte den Ersten Weltkrieg in der Tradition der Kriege des 19. Jahrhunderts. Aus den Gefallenen wurden unsterbliche Märtyrer der Nation, ihr Tod das wertvollste Opfer auf dem Altar des Vaterlandes und Vermächtnis für die Zukunft. „Geben wir mit diesem Denkmal“, appellierte Tomov, dem „Emblem bulgarischer Heimatliebe, bulgarischer Entsagung, Opferbereitschaft und Tapferkeit [zukünftigen, Anm. d. Verf.] Generationen ein ewiges erbauliches Beispiel für vaterländische Pflichterfüllung“" ${ }^{33}$ Der Krieg bekam seinen Platz im kollektiven Gedächtnis und in der symbolischen Landschaft Bulgariens. Neben der Repräsentation der offiziellen Vergangenheitspolitik aber erfüllte das Denkmal auch eine sozial-karitative Aufgabe, derer sich die Bauernpartei durchaus bewusst war. Das Denkmal des „Unbekannten Soldaten“ war nicht nur ein ,site of memory“, sondern ebenso, wie der britische Historiker Jay M. Winter bei der Analyse von Weltkriegsdenkmälern betont hat, ,a place, where people could grieve“. ${ }^{34}$ Mit dem Denkmal als Ort der Erinnerung und der Trauer reagierten die Agrarier auf die Verlusterfahrung vieler Familien, die nicht ,,an den verstreuten Gräbern Blumen niederlegen und sich in ihrem Unglück wenigstens ein bisschen trösten können“. „Geben auch wir den Vätern, Müttern, Witwen und Waisen wenigstens ein Grab für ihre unvergesslichen Verluste“, ${ }^{35}$ hatte Tomov gefordert. Die Konstruktion einer nationalen Trauer- und Solidargemeinschaft entsprach dem Bedürfnis einer Bevölkerung, die geschockt vom Sterben den Sinn für den Tod gleichwohl im Nationalen suchte. Die Gleichzeitigkeit von romantischem Vereinigungsnationalismus und tiefer Kriegsablehnung, die die Erinnerungspolitik der neuen Regierung leitete, befand sich durchaus in Einklang mit einer Stimmung, die bereits in den Kriegsjahren, u.a. in Feldpostbriefen, deutlich geworden war. Dort, so hat die bulgarische Historikerin Snežana Dimitrova gezeigt, wurde die Besetzung Makedoniens einerseits begeistert geschildert - „,500 Jahre war unsere liebe Mutter Makedonien eine schwarze Sklavin, in Ketten gefesselt $[\ldots]$, nun haben wir unsere liebe Mutter befreit, und mit eiserner Brust werden wir sie beschützen und sind bereit, für sie zu sterben." Anderer-

32 Ebd.

33 Ebd.

34 Winter 1995, 79.

35 CDIA, fond 173 , op. 3, a. e. 3185 , list 2. 
seits tauchten Sätze auf wie: „Es reicht mir mit diesen Kriegen.“ ${ }^{“ 36}$ Die Agrarier fügten die Kriegsmüdigkeit und das Trauerbedürfnis in ihrem Denkmalprojekt zusammen, mit dem sie, darüber hinaus, an die europäische Kultur der Kriegserinnerung nach 1918 anknüpften. So waren in den meisten kriegsbeteiligten Staaten, häufig in den Siegernationen, nach Kriegsende „Denkmäler des unbekannten Soldaten“ als Antwort auf die über den ganzen Kontinent verstreuten anonymen Massengräber entstanden. ${ }^{37}$ Obwohl Bulgarien zu den Verlierern gehörte, knüpfte das Land an diese Entwicklung nicht zuletzt mit dem Ziel an, als geläuterte Nation in den Schoß der europäischen Zivilisation zurückzukehren. „Alle zivilisierten Staaten“, betonte Tomov,

haben an ihren bedeutendsten Plätzen [...] Denkmäler für [...] unbekannte Krieger errichtet [...], das Bild, das wir unter dem Triumphbogen in Paris sehen, [...] und in Potsdam bei Berlin ist das Bild der [...] zivilisierten Welt. ${ }^{38}$

Die staatlichen Pläne für die Kriegserinnerung kamen jedoch über das Anfangsstadium nicht hinaus. Weder wurde das „Denkmal für den unbekannten Soldaten" gebaut noch ein ursprünglich angedachter nationaler Trauertag ausgerufen. Die kurze Lebensdauer der Stambolijski-Regierung verhinderte zunächst einen Gefallenenkult, wie er in Europa zum Markenzeichen der Kriegserinnerung wurde. Ein halbes Jahr nach dem Vorschlag Tomovs war die Regierung gestürzt. Aber auch die gesellschaftlichen Vereine brachten keine nennenswerten Gedenkinitiativen hervor. Während in den anderen Staaten Europas die Kriegsveteranen- und Offiziersverbände den nationalen Totenkult initiierten, entstanden in Bulgarien bis 1923 weder Heldenhaine noch Soldatenfriedhöfe oder eine Organisation ähnlich dem deutschen „Volksbund deutscher Kriegsgräberfürsorge“. Am Willen der Veteranen fehlte es keineswegs. Die Ursache für die eingeschränkte Erinnerungsarbeit waren vielmehr die Restriktionen im 1922 in Kraft getretenen „Gesetz über die Schulen und Vereine“, die, indem sie die Vereine unter die Kontrolle des Innenministeriums zwangen, die Freiheit dieser traditionellen Trägerschicht erheblich beschnitten. ${ }^{39}$ Zwar war die Bauernpartei durchaus gewillt, Zugeständnisse an die

36 So zitiert bei Dimitrova 2000, 2, 206-227, $216 f f$. Siehe auch dies. 1998, 301-374.

37 Zur europäischen Kriegsdenkmalkultur u. a. bei Mosse 1980, 241-261. Koselleck 1994, 9-20; Jeismann / Westheder 1994, 23-50; King 1998; Moriaty 1999, 4, 653-662; Sherman 1994, 186-211.

38 CDIA, fond 173 , op. 3, a. e. 3185 , list 2.

39 Dăržaven vestnik XLIV, 1922, 86: 1. Nach diesem Gesetz waren alle Vereinigungen und Komitees des Landes verpflichtet, ihre Unterlagen beim Innenministerium bestätigen zu lassen. Wurde das Statut ohne Beanstandung akzeptiert, musste das Komitee jährlich einen Rechenschafts- und Finanzbericht einreichen. Außer den Veteranenverbänden des „Befreiungskrieges“, deren Zahl arg geschrumpft war, war es keinem Militärverein gestattet, Beziehungen zum Kriegsministerium zu unterhalten. Durch das Gesetz verfügt das Archiv des Innenministeriums heute über eine nahezu lückenlose Auflistung von Denkmalkomitees und historischen Vereinen. 
Kriegsdeutungen der Veteranen zu machen; die Deutungsmacht aber sollte allein der staatlichen Vergangenheitspolitik vorbehalten bleiben.

Trotz der staatlichen Restriktionen entstanden auch in Bulgarien Kriegsdenkmäler nach jener „optischen Signatur“"40, die Reinhart Koselleck für den modernen Denkmalkult ausgemacht hat. Noch während des Krieges, in weitaus größerem Maße aber nach 1918 wurden im ganzen Land jene Obelisken, Pyramiden und Gedenktafeln errichtet, die auf dem gesamten Kontinent vom Bedürfnis zeugten, den Sinn des Todes im vaterländischen Opfertod zu verorten. ${ }^{41}$ Zweifelsohne entsprachen diese, nicht von Vereinen, sondern von den Familien und Heimatgemeinden errichteten Denkmäler zuerst einem anthropologischen Grundbedürfnis nach Trauer- und Verlustbewältigung, das jedoch angesichts der entgrenzten Kriegsgewalterfahrung über das private Leid hinaus nach einer explizit kollektiven Sinngebung verlangte. Den Hinterbliebenen, häufig privaten Sponsoren der Denkmäler, war es wichtig, ihren Toten ein Grab und sich selbst einen Ort des Gedenkens, aber auch eine „höhere“ Erklärung für den Verlust zu geben. Die lokalen Kriegsdenkmäler waren nicht nur Zeugnisse einer privaten Trauerarbeit. Auf Friedhöfen ${ }^{42}$, an Schulen ${ }^{43}$ oder auf dem Dorfplatz ${ }^{44}$ in gemeinschaftlicher Aktion errichtet, verbanden sie private grief and public remembrance. ${ }^{45}$ Der Tag, an dem der Toten gedacht wurde, war nicht dem privaten Lebenszyklus (Geburts- oder Sterbetag) entnommen, sondern meist ein religiöser oder volkskultureller Feiertag, an dem sich die Gemeinde zum Gottesdienst vor dem Denkmal versammelte. Besonders populär waren die orthodoxen Totengedenktage, die „zadušnici“ im November, Heiligentage wie der Tag des Erzengel Michail am 8. November (Archangelovden), der Tag des Heiligen Demetrius am 26. Oktober (Dimitrovden) und der Tag des Heiligen Georg am 6. Mai (Georgovden), Ostern (văznesenie gospodne) oder der 24. Mai, der Tag der Slawenapostel Kyrill und Method. Teilweise wurde wie in Orjachovica der Tag der Dorfver-

40 Koselleck 1979, 255-276, 275.

41 Das nationale Institut für Kulturdenkmäler in Sofia verfügt über die umfangreichste Liste aller in Bulgarien errichteten Kriegsdenkmäler. Der Fond ,vojniški pametnici“ ist - in den 1930er-Jahren vom Kriegsministerium angelegt - nach 1944 bis zur Auflösung der Denkmalabteilung weitergeführt worden. Er enthält nahezu 2.000 Denkmäler, geordnet nach Entstehungsjahr, Initiatoren, Standort, Kosten, Gedenktag. Archiv na nacionalen institut za pametnici na kulturata (weiter: Archiv na NIzPnK), fond vojniški pametnici.

42 So u.a. in Asparuchovo 1920 (Burgaski oblast), Basarovo 1921 (Rusenka okolija), Vjaglevšti 1918 (Tărnovski oblast). Archiv na NIzPnK, fond vojniški pametnici, passim.

43 So u.a. in Benkovski 1922 (Gebiet Stara Zagora), Vătren 1921 (Gebiet Pazardžik), Glavaci 1920 (Gebiet Vraca). Ebd.

44 So u.a. in Damjanovo 1919 (Gebiet Gabrovo), Divotino 1919 (Gebiet Sofia), Željava 1920 (Gebiet Sofia). Ebd.

45 Moriaty 1997, 125-142. 
sammlung (selski săbor, 14. September) für das gemeinsame Gedenken genutzt. ${ }^{46}$

Die Standorte, die kollektiven Gedenkrituale und damit die Partizipation auch derer, die nicht zur persönlich betroffenen Trauergemeinschaft gehörten, bestimmten die private Trauer und öffentliche Erinnerung. Im lokalen Kriegerdenkmal des Ersten Weltkrieges wurde der individuelle Tod in einen kollektiven Sinn eingebettet, der auch die Trauer zu einer gemeinschaftlichen Angelegenheit und Verpflichtung machte. So wie der Tod im Schützengraben ein nationaler war, war auch die Trauer in der nationalen Deutung aufgehoben. Nicht die Entpersonalisierung oder Anonymisierung des Todes, sondern die im Denkmalprojekt erfahrbare Verbindung von individuellem Schicksal und nationalem Sinn prägte die Wirkungsmacht der Kriegserinnerung im lokalen Raum. Der vaterländische Opfertod war dabei die moderne, auf einem Konsens zwischen Staat und Gesellschaft basierende Sinnstiftung. „It is important to emphazise that the memorials rarely challenged official interpretations of the war" ${ }^{47}$ betonte Catherine Moriaty für die Kriegserinnerung in Großbritannien. Selbiger Befund trifft ebenso auf die „Verlierernation“ Bulgarien zu, dominierte doch auch hier in der lokalen Gedenkkultur nach 1918 die nationale Deutung weitgehend frei von staatlichen Vorgaben.

Während der Regierungszeit der Agrarier und auch in den Jahren danach nahm die Politik weder Einfluss auf das Aussehen der lokalen Denkmäler, die Festlegung der Gedenktage, noch stieß sie sich, wie in den kommenden Jahren, an der hohen Emotionalität volkskulturell-religiösen Gedenkens. Bis 1923 wurde der Toten selten an Staatsfeiertagen gedacht. Nur vereinzelt wie im Dorf Negovanovci (Gebiet Vidin), wo das Denkmal allerdings schon 1912 entstanden war, war der 3. März, der „San Stefano-Tag“, alternativ zum Tag des Heiligen Georg (6. Mai) als Trauertag festgelegt worden. ${ }^{48}$ Finanziell unterstützte der Staat den lokalen Denkmalbau allenfalls sporadisch. Als z. B. 1922 ein Komitee aus Gorna-Banja, einem Dorf nahe Sofia, den Antrag auf Schenkung von sechzehn Eisenplatten aus Staatsbesitz zur Verzierung eines Kriegerdenkmals stellte, wurde dem Anliegen vom Ministerrat im November 1922 stattgegeben, nachdem der Minister für Eisenbahn, Post und Telegrafen auf die Bedeutung des Denkmalbaus hingewiesen hatte. ${ }^{49}$ Erst als in den 1930er-Jahren die Trauer einem staatlich gelenkten nationalistischen Heldenkult Platz machte, alimentierte der Staat den Denkmalbau und veränderte die

46 Archiv na NIzPnK, fond vojniški pametnici, papka VIII.

47 Moriaty 1997, 126.

48 Archiv na NIzPnK, fond vojniški pametnici, papka VIII.

49 CDIA, fond 173, op. 3, a. e. 3260, list 1-4 u. a. Erklärung zum Gesetzesprojekt einer Schenkung von 16 Eisenplatten für das Denkmalkomitee für die Gefallenen der Kriege 1912-1913 und 1915-1918 in Gorna-Banja vom 16. Dezember 1922 (izloženie na motivite kăm proekto-rešenieto za opuštane darom na komiteta za văzdigane pametnik v s. Gorna-Banja, Sofijska okolija, na zaginalite prez vojnite 1912-1913 i 1915-1918 godini šestnadeset platna pleteni železa). 
Gedenktage. Zukünftig waren die Gefallenen vermehrt an Staatsfeiertagen oder am Tag des Heiligen Georg, der in der Königsdiktatur als „,Tag der bulgarischen Tapferkeit“ Teil offizieller Symbolpolitik geworden war, zu ehren.

Trotz der Übereinstimmung zwischen der Kriegsdeutung der Bauernpartei und dem Erinnerungsbedürfnis eines großen Teils der bulgarischen Bevölkerung konnten die Agrarier die lokale Denkmalkultur nicht für ihre Imagepolitik verbuchen. Bulgarische Kriegsdenkmäler waren bis 1923 aus dem gesellschaftlichen Begehren nach Kriegsverarbeitung entstandene Sinnstiftungen der Überlebenden, die der offiziellen Vergangenheitspolitik zwar nicht zuwiderliefen, die sich dem staatlichen Einfluss jedoch auch entzogen. Beide existierten nebeneinander. Die Bauernpartei hatte eine Kriegserinnerung favorisiert, die kein territoriales Ideal, wohl aber eine im Leid vereinte nationale Schicksalsgemeinschaft beschwor. Es war eine explizit vaterländische Opferbereitschaft, die dem Massensterben Sinn verlieh, der Gegenwart eine Berechtigung und der Zukunft Zuversicht gab. Nation, Vaterland und patriotische Pflichterfuillung waren die Einheits- und Integrationscodes einer Partei, die sich politisch der Überwindung nationaler Beschränktheit verschrieben hatte und sich der emotionalen Macht des Nationalen schließlich nicht entziehen konnte.

\section{Zusammenfassung}

1920 übernahm die Bauernpartei die politische Macht in einem vom Krieg traumatisierten Land. Ihr hoher Reformanspruch scheiterte an innen- und außenpolitischen Widerständen, am Terror der IMRO, an der internationalen Isolation der „Verlierernation“, an der andauernden Wirtschaftskrise, aber auch an den diktatorischen Mitteln, mit denen Stambolijski seine Macht erhalten wollte. Vergangenheitspolitisch befand sich der BZNS von Beginn an in einem Dilemma zwischen der Abkehr von den kollektiven Sinnangeboten der Vergangenheit und dem Sicherheitsbedürfnis der Bevölkerung. Wie konnte die neue Politik balkanischer Verständigung in einer Gesellschaft durchgesetzt werden, die über Jahre hinweg Feindbilder und Stereotype vom ,heimtückischen Nachbarn" verinnerlicht hatte? Mit welchem Sinnangebot waren die Wunden des Massensterbens zu heilen? 1918 war nicht nur der Erste Weltkrieg verloren, sondern mit ihm das Deutungsnetz nationaler Identität und Werte. Indem sie den Kriegsnationalismus ablehnte, die Nation als Fluchtpunkt kollektiver Identität aber aufrechterhielt, wählte die Bauernpartei eine janusköpfige Krisenstrategie. Auf der Suche nach einer kriegsentlastenden bulgarischen Identität wurden die Kollektivchiffren des „nationalen Ideals“ und „Bulgarentums“ von militarisierten Bedeutungen wie "Territorium“ und „Kampf“" befreit und mit den Codewörtern „Kultur“ und „Bildung“ belegt. Anstelle der Kriegsnation sollte die Vorstellung einer Kulturnation Sicherheit und Orientierung vermitteln. Die Bauernpartei inszenierte den „neuen Bulgaren" mit traditionellen Erinnerungen und Images von den Mittelaltermythen 
bis zur „Wiedergeburt“, die bereits während und vor der Kriegsepoche zu den symbolischen Stützen kollektiver Selbstvergewisserung gehört hatten. Der 24. Mai war der „Tag des bulgarischen Schrifttums“, an dem Eigenbilder kultureller Superiorität teilweise verbrämt, teilweise aber auch offen formuliert werden konnten. Die Agrarier bedienten sich am vergangenheits- und bildungspolitischen Repertoire der Jahrhundertwende und waren mit ihrer Symbolpolitik so kompatibel, dass auch die folgenden autoritären Regierungen bedenkenlos daran anknüpfen konnten. Die Vergangenheitspolitik der Bauernpartei war von Anpassungswillen und tiefem Traditionsbewusstsein geprägt. Der krisengeschüttelte, traumatisierte Zustand Nachkriegsbulgariens erklärt den symbolpolitischen Konservatismus der Agrarier zu großen Teilen, aber nicht ausschließlich. Selbst ohne diese Krise wäre die Erosion des Nationalen für die Agrarier von zweifelhaftem Nutzen gewesen. Um den politischen Nutzen nationaler Erinnerungen dürften auch die Agrarier gewusst haben, die sich damit bewusst vom Internationalismus ihrer schärfsten Konkurrenz, der Kommunistischen Partei, abgrenzten. Der Rückgriff auf die Nation konnte die Integrationsdefizite des eigenen Gesellschaftsentwurfs übertünchen. Zur Nation hatte die Bauernpartei keine Alternative. Omarčevskis kulturnationalistische Vergangenheitspolitik bot lediglich eine andere Ausdeutung nationaler Überlegenheit, die zu keiner Zeit infrage gestellt war. Die Jahre der Bauernpartei waren kein erinnerungskultureller Neuanfang, sondern geprägt von der Reaffirmation tradierter Identitätsparadigmen, die von den politischen Vorgängern ebenso funktionalisiert worden waren, wie sie später von den Nachfolgern Stambolijskis eingesetzt werden sollten.

\section{Literaturverzeichnis}

\section{Quellen:}

Archiv na nacionalen institut za pametnici na kulturata. Sofija (Archiv na NIzPnK)

Fond vojniški pametnici.

Centralen dăržaven istoričeski archiv. Sofija (CDIA).

Fond 173 Narodno săbranie.

Fond 177 Ministerstvo na narodnata prosveta.

Bălgarski voenen almanach. Sofija 1926.

Dăržaven vestnik. Sofija 1922.

Omarčevski, Stojan, 1920: Zemedelskija săjuz i učlilisteto. Sofija.

Otčet na ministra na narodnoto prosveštenie Stojan Omarčevski za dejnostta na ministerstvoto ot 20 maj 1920 go 1 april 1921 god. Sofija 1921.

Sv. Sv. Kiril i Metodij 24/11 maj 1921 god. Izdanie na ministerstvoto na narodnoto prosveštenie. Sofija 1921.

Tadžer, Ž., 1922: Nova Bălgarija. Ministerite i ministerstvata. Sofija. 
Učilišten pregled. Sofija 1921.

Zemedelska Bălgarija. Sofija 1922.

Zlatnijat almanach na Bălgarija. Sofija 1938.

\section{Monographien, Artikel:}

Bell, John D., 1977: Peasants in Power. Alexander Stamboliski and the Bulgarian Agrarian National Union, 1899-1923. Princeton.

Božkov, Ljubomir / Ninov, Stojan, 1980: The Historical Path of the Bulgarian Agrarian Party. Sofija.

Crampton, Richard J., 1993: A Short History of Modern Bulgaria. Cambridge.

Dimitrova, Snežana, 1998: Văzstanovjavane ... Reperacii ... Garancii ... Francija i balkanskite slavjanski dăržavi, Septemvri 1918 - Januari 1920. Blagoevgrad.

Dimitrova, Snežana, 2000: „,Mojata zemja“, ,Mojata scena“, ,Moeto probuždane‘: Figurativnite preživjavanija na prirodata po vreme na goljamata vojna (1914-1918) i bălgarskata modernist", in: Kritika i Humanism 9(2000), 2, 206-227.

Dimitrova, Snežana, 2002: „,My War is not Your War': The Bulgarian Debate on the Great War", in: Rethinking History 6(2002), 1, 1-9.

Fussell, Paul, 1975: The Great War and Modern Memory. Oxford.

Hirschfeld, Gerhard / Langewiesche, Dieter / Krumeich, Gerd (Hrsg.), 1997: Kriegserfahrungen. Studien zur Sozial und Mentalitätsgeschichte des Ersten Weltkriegs. Essen.

Jeismann, Michael / Westheder, Rolf, 1994: „Wofür stirbt der Bürger? Nationaler Totenkult und Staatsbürgertum in Deutschland und Frankreich seit der Französischen Revolution“, in: Koselleck, Reinhart / Jeismann, Michael (Hrsg.): Der politische Totenkult. Kriegerdenkmäler in der Moderne. München, 23-50.

King, Alex, 1998: Memorials of the Great War in Britain: The Symbolism and Politics of Remembrance. Oxford, New York.

Koselleck, Reinhart, 1979: „Kriegerdenkmale als Identitätsstiftungen der Überlebenden“, in: Marquardt, Odo / Stierle, Karlheinz (Hrsg.): Identität. München, 255-276.

Koselleck, Reinhart, 1994: „Einleitung“, in: Koselleck, Reinhart / Jeismann, Michael (Hrsg.): Der politische Totenkult. Kriegerdenkmäler in der Moderne. München, 9-20.

Mišev, Dimităr, 1921: „Slavjanstvoto i Bălgarija“, in: Sv. Sv. Kiril i Metodij 24/11 maj 1921 god. Izdanie na ministerstvoto na narodnoto prosveštenie. Sofija, 9ff.

Moriaty, Catherine, 1997: „Private Grief and Public Remembrance: British First World War Memorials“, in: Evans, Martin / Lunn, Ken (Hrsg.): War and Memory in the Twentieth Century. Oxford, 125-142.

Moriaty, Catherine, 1999: „The Material Culture of Great War Remembrance“, in: Journal of Contemporary History 34(1999) 4, 653-662.

Mosse, George L., 1980: „Soldatenfriedhöfe und nationale Wiedergeburt. Der Gefallenenkult in Deutschland“, in: Vondung, Klaus (Hrsg.): Kriegserlebnis: Der Erste Weltkrieg in der literarischen Gestaltung und symbolischen Deutung der Nationen. Göttingen, 241-261. 
Mosse, George L., 1990: Fallen Soldiers. Reshaping the Memory of the World Wars. New York.

Rothschild, Joseph, 1990: East Central Europe between the Two World Wars. Seattle, London.

Schivelbusch, Wolfgang, 2001: Die Kultur der Niederlage. Der amerikanische Süden 1865, Frankreich 1871, Deutschland 1918. Berlin.

Sherman, Daniel J., 1994: „Art, Commerce, and the Production of Memory in France after World War I“, in: Gillis, John R. (Hrsg.): Commemorations. The Politics of $\mathrm{Na}$ tional Identity. Princeton, 186-211.

Stambolijski, Aleksandăr, 1980: Život, delo, zaveti. Sofija.

Winter, Jay, 1988: The Experience of World War I. London.

Winter, Jay, 1995: Sites of Memory, Sites of Mourning. The Great War in European Cultural History. Cambridge.

Winter, Jay, et. al. (Hrsg.), 1999: War and Remembrance in the Twentieth Century. Cambridge. 
